\title{
Autophagy in Spinocerebellar ataxia type 2, a dysregulated pathway, and a target for therapy
}

\author{
Adriana Marcelo iD $^{1,2,3,4}$, Inês T. Afonso ${ }^{1}$, Ricardo Afonso-Reis ${ }^{1,4}$, David V. C. Brito (iD ${ }^{1}$, Rafael G. Costa ${ }^{1,4}$, Ana Rosa ${ }^{1}$, \\ João Alves-Cruzeiro ${ }^{3}$, Benedita Ferreira ${ }^{1,4}$, Carina Henriques ${ }^{3}$, Rui J. Nobre ${ }^{3}$, Carlos A. Matos (iD ${ }^{1,4}$, Luís Pereira de Almeida ${ }^{3,5}$ and \\ Clévio Nóbrega (iD) ${ }^{1,4,6 \times}$
}

(c) The Author(s) 2021

\begin{abstract}
Spinocerebellar ataxia type 2 (SCA2) is an incurable and genetic neurodegenerative disorder. The disease is characterized by progressive degeneration of several brain regions, resulting in severe motor and non-motor clinical manifestations. The mutation causing SCA2 disease is an abnormal expansion of CAG trinucleotide repeats in the ATXN2 gene, leading to a toxic expanded polyglutamine segment in the translated ataxin-2 protein. While the genetic cause is well established, the exact mechanisms behind neuronal death induced by mutant ataxin-2 are not yet completely understood. Thus, the goal of this study is to investigate the role of autophagy in SCA2 pathogenesis and investigate its suitability as a target for therapeutic intervention. For that, we developed and characterized a new striatal lentiviral mouse model that resembled several neuropathological hallmarks observed in SCA2 disease, including formation of aggregates, neuronal marker loss, cell death and neuroinflammation. In this new model, we analyzed autophagic markers, which were also analyzed in a SCA2 cellular model and in human post-mortem brain samples. Our results showed altered levels of SQSTM1 and LC3B in cells and tissues expressing mutant ataxin-2. Moreover, an abnormal accumulation of these markers was detected in SCA2 patients' striatum and cerebellum. Importantly, the molecular activation of autophagy, using the compound cordycepin, mitigated the phenotypic alterations observed in disease models. Overall, our study suggests an important role for autophagy in the context of SCA2 pathology, proposing that targeting this pathway could be a potential target to treat SCA2 patients.
\end{abstract}

Cell Death and Disease (2021)12:1117; https://doi.org/10.1038/s41419-021-04404-1

\section{INTRODUCTION}

Spinocerebellar ataxia type 2 (SCA2) is a rare and fatal dominantlyinherited polyglutamine (polyQ) disease [1, 2], being caused by abnormal repeats of the triplet CAG in the ATXN2 gene, resulting in an expanded polyQ chain in the ataxin-2 protein [3, 4]. In healthy individuals, the protein carries between 13-31 glutamines (Q), while repeats above that threshold lead to disease development [5]. The mutation results in progressive and widespread degeneration of different neuronal populations, including the cerebellum, pons, basal ganglia, thalamus and midbrain [6]. The clinical manifestations include motor dysfunctions such as progressive gait ataxia, dysarthria, postural tremors and bradykinesia, as well as visual impairments and cognitive/psychiatric symptoms $[7,8]$. Currently, SCA2 patients rely only on pharmacological compounds to relief symptomatology.

Understanding the molecular mechanisms underlying SCA2 pathogenesis is essential to find new targets and an efficient therapy for this incurable disorder. The development and characterization of several SCA2 cellular and animal models, already provided some hints in the events that lead to neuronal death. The disease-causing polyQ expansion induces conformational alterations in the native ataxin-2 structure, resulting in formation of insoluble fibrillar bodies that accumulate as aggregates inside neuronal cells $[9,10]$. Although the role of aggregation in SCA2 pathogenesis remains unresolved, analysis of patients' post-mortem brain samples revealed a positive correlation between the presence of mutant ataxin- 2 inclusions and the severity/extension of neuronal degeneration [11]. Moreover, processes such as oxidative stress, disruption of cell signaling and disturbance of calcium homeostasis have been shown to be involved in SCA2 pathogenesis [12]. Despite several advances, the exact mechanisms through each mutant ataxin-2 leads to death of neurons are still controversial and need to be further elucidated.

As mutant polyQ proteins form large multi-protein inclusions, protein degradation systems are essential to maintain cellular homeostasis. An important protein clearance mechanism is autophagy, a key pathway implicated in other polyQ diseases pathogenesis [13]. Autophagic markers, such as LC3B (microtubule-associated protein 1 light-chain 3B) or SQSTM1 (sequestosome 1), have been observed in polyQ pathological aggregates suggesting autophagy impairment $[14,15]$. In line with this, we previously demonstrated that pharmacologic activation of

\footnotetext{
${ }^{1} \mathrm{ABC}-\mathrm{Rl}$, Algarve Biomedical Center Research Institute, Algarve Biomedical Center, Faro, Portugal. ${ }^{2}$ PhD Program in Biomedical Sciences, Faculdade de Medicina e Ciências Biomédicas, Universidade do Algarve, Faro, Portugal. ${ }^{3}$ Center for Neuroscience and Cell Biology (CNC), University of Coimbra, Coimbra, Portugal. ${ }^{4}$ Faculty of Medicine and Biomedical Sciences, University of Algarve, Faro, Portugal. ${ }^{5}$ Faculty of Pharmacy, University of Coimbra, Coimbra, Portugal. ${ }^{6}$ Champalimaud Research Program, Champalimaud

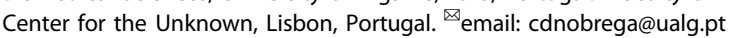


autophagy, using the compound cordycepin, reduced the neuropathological and motor deficits in mouse models of Machado-Joseph disease (MJD) (ref. [16]).

In SCA2, the analysis of patients' mRNA levels revealed increased levels of the autophagic protein WDFY3 (WD repeat and FYVE domain containing 3) (ref. [17]). Also, STAU1 (staufen 1) protein was found to be augmented in SCA2 patients-derived fibroblasts due to autophagy dysfunction [18]. However, there is a lack of studies investigating the presence of autophagy markers in the context of SCA2, or in the study of autophagy activation as a therapeutic strategy. Therefore, in this work we aimed at evaluating the autophagic process in a new mouse model of SCA2, as well as cellular models and patients' brain tissues. We found that SQSTM1 and LC3B levels are altered in SCA2, suggesting a dysregulation of autophagy pathway. Importantly, molecular activation of autophagy, through cordycepin, reduced mutant ataxin- 2 aggregation in the SCA2 lentiviral mouse model.

\section{MATERIAL AND METHODS \\ Animals}

Adult C57BL/6 mice, breed in the animal house facility of the Algarve Biomedical Center Research Institute of the University of Algarve, were used in this work. All animals were housed in a temperature-controlled room on a $12 \mathrm{~h}$ light-12 $\mathrm{h}$ dark cycle. Food and water were provided ad libitum. The number of animals used in each experiment was based in previous studies and in the recommendation to reduce the number of animals used in experimentation. The experiments were carried out in accordance with the European Community directive (86/609/EEC) for the care and use of laboratory animals. The researchers received certified training (FELASA course) and approval to perform the experiments from the Portuguese authorities (Direcção Geral de Alimentação e Veterinária) in the project Neuropath (421/2019).

\section{Lentiviral vectors}

Lentiviral particles (LVs) encoding for human full-length ataxin-2 with either normal polyQ segment (23Q - Atx2WT) or mutant polyQ segment ( $80 \mathrm{Q}$ or $104 \mathrm{Q}-$ Atx2MUT) under the control of the mouse phosphoglycerate kinase 1 (PGK) promoter were produced in HEK (human embryonic kidney) 293T cells using a four-plasmid system, described elsewhere [19] and quantified by p24 antigen ELISA (RETROtek, Gentaur, France) by ViraVector facility, University of Coimbra. Additionally, the lentiviral vectors titer was quantified using the Lentivirus qPCR Titer Kit (Applied Biological Materials Inc, Vancouver, Canada), allowing the determination of the virus titer in infectious units per $\mathrm{ml}(\mathrm{IU} / \mathrm{ml})$.

\section{Striatal lentiviral mouse model}

To develop the SCA2 lentiviral mice, concentrated LVs viral stocks encoding for Atx2WT or Atx2MUT were thawed on ice and homogenized. Adult (8/10-week-old) C57BL/6 mice (both males and females) were anesthetized through intraperitoneal injection (IP) of a mixture of ketamine $\left(75 \mathrm{mg} / \mathrm{kg}\right.$, Nimatek, Dechra) with medetomidine $\left(0.75 \mathrm{mg} / \mathrm{kg}\right.$, DOMTOR ${ }^{\circledR}$, Esteve). LVs were administered through a stereotaxic surgery into the left (Atx2WT) or the right (Atx2MUT) hemisphere of the striatum according to the following brain coordinates relative to bregma: antero-posterior $(+0.6 \mathrm{~mm})$; lateral $( \pm 1.8 \mathrm{~mm})$; ventral $(-3.3 \mathrm{~mm})$. A concentration of $400.000 \mathrm{ng}$ of p24 antigen $(2 \mathrm{E} 10 \mathrm{IU} / \mu \mathrm{l})$ in $2 \mu \mathrm{l} \mathrm{of} \mathrm{LVs}$ were injected at a rate of $0.25 \mu \mathrm{l} / \mathrm{min}$, by means of an automatic injector (Stoelting Co., Wood Dale, IL, USA), into mice striatum using a 34-gauge blunt-tip needle linked to a Hamilton syringe (Hamilton, Reno, NV, USA). Four, eight and twelve weeks after surgery, mice were sacrificed for posterior analysis.

\section{Tissue preparation}

For all immunohistochemical assays, animals received an anesthesia (mixture described above) overdose, followed by a transcardial perfusion with $4 \%$ paraformaldehyde (PFA) solution SigmaAldrich). Upon removal, brains were left $24 \mathrm{~h}$ in $4 \%$ PFA solution, dehydrated in a $30 \%$ sucrose $/ 0.1 \mathrm{M}$ phosphate buffer solution (PBS) for $48 \mathrm{~h}$ and cryoprotected at $-80^{\circ} \mathrm{C}$ degrees. Posteriorly, coronal brain slices of $20 \mu \mathrm{m}$ thickness were obtained using a cryostat (Cryostar NX50, ThermoFisher Scientific) and stored in freefloating PBS with $0.05 \mu \mathrm{m}$ sodium azide solution at $4^{\circ} \mathrm{C}$. For western blotting procedures, animals received an anesthesia overdose, followed by cervical dislocation. Brains were removed and striatal punches, using a Harris Core pen with $2.5 \mathrm{~mm}$ diameter (Ted Pella Inc., Redding, California, USA), were collected and stored at $-80^{\circ} \mathrm{C}$ until subsequent processing.

\section{Behavioral analysis}

For evaluation of exploratory behavior, mice stereotaxically injected with LVs, encoding for either Atx2WT or Atx2MUT in the right striatum hemisphere, were subjected to open field at 4- and 8-weeks post-surgery. Mice were placed in an activity cage (Panlab, Barcelona, Spain) with $50 \times 50 \mathrm{~cm}$ arena and $50 \mathrm{~cm}$-high walls for $40 \mathrm{~min}$ and movement activity was recorded using the Anti-Track System (Panlab, Barcelona, Spain). The collected data was blindly analyzed for the last 30min of activity. Experimental tests were performed in the same room, with lights turned off and after 60 min minimum of space acclimatization.

\section{Neuroblastoma cell culture and transfection}

Mouse neuroblastoma line-derived cells (Neuro-2A), obtained from the American Type Culture Collection cell biology bank (CCL-131), were cultured in Dulbecco's modified Eagle's medium (DMEM) supplemented with $10 \%$ fetal bovine serum (FBS), $100 \mathrm{U} / \mathrm{ml}$ penicillin and $100 \mathrm{mg} / \mathrm{ml}$ streptomycin (Gibco) at $37^{\circ} \mathrm{C}$ in $5 \% \mathrm{CO} /$ air atmosphere. These cells are routinely tested for mycoplasma contamination. For transfection assays, complexes of $3 \mu \mathrm{l} \mathrm{PEl} \mathrm{solution} \mathrm{(polyethylenimine} 1 \mathrm{mg} / \mathrm{ml}$, Tebu-bio) with 500 ng of DNA plasmids were formed in $10 \mu \mathrm{l}$ DMEM without supplementation and added to cells previously cultured in 12-well plates for $24 \mathrm{~h}$. Transfection reagents were left in cells for a 48h-period incubation, following cells harvest for immunocytochemistry or western blot assays. The following DNA constructs were used: human full-length ataxin-2 with normal polyQ segment (23Q - Atx2WT) and mutant polyQ segment (80Q Atx2MUT); human full-length ataxin-2 with an EGFP (enhanced green fluorescent protein) tag carrying normal polyQ segment (22Q - Atx2-Q22) or mutant polyQ segment (58Q - Atx2-Q58 or 104Q - Atx2-Q104) (ref. [20]); ataxin-2 truncated form with an EGFP tag carrying normal polyQ segment (22Q - Atx2-Q22T) or mutant polyQ segment (58Q - Atx2-Q58T); ptfLC3RFP-GFP (Addgene \#21074) (ref. [21]). For chloroquine (ChQ) experiments, cells were treated $6 \mathrm{~h}$ before harvest with $100 \mu \mathrm{M}$ of $1 \mathrm{mg} / \mathrm{ml}$ ChQ (Sigma) solution in DMSO. Starvation condition was induced by replacing cells culture medium with Hanks' Balanced Salt Solution (HBSS 1X) (Gibco) supplemented with $1.8 \mathrm{~g} / \mathrm{L}$ of glucose $6 \mathrm{~h}$ before harvest.

\section{Cordycepin treatment - in vitro and in vivo studies}

For in vitro studies, Neuro-2A cells transfected with EGFP Atx2-Q104 construct or ptfLC3-RFP-GFP were treated with $20 \mu \mathrm{M}$ cordycepin (3'deoxyadenosine, $300 \mathrm{mg} / \mathrm{ml}$ in DMSO, TargetMol) for $48 \mathrm{~h}$ and harvested for direct fluorescence analysis.

For in vivo experiments, Adult (8/10-week-old) C57BL/6 mice (both males and females) were stereotaxically injected with LVs, encoding for Atx2MUT in the right striatum hemisphere. For the long-term treatment, cordycepin administration began one week after surgery. Treated mice were administered an IP injection of $20 \mathrm{mg} / \mathrm{Kg}$ of cordycepin in DMSO-NaCl $0.1 \%$ solution, while control group received an IP injection of the vehicle ( $\mathrm{NaCl} 0.1 \%), 2 x$ per week for five weeks, $3 x$ per week during following four weeks and $5 x$ per week during final three weeks (the animals were randomly distributed in each group after the stereotaxic injection). The concentrations and timepoints were defined considering our previous study using cordycepin in in vivo experiments [16]. After final treatment week, mice were sacrificed for analysis. In the short-term treatment, the animals received either an IP injection of $20 \mathrm{mg} / \mathrm{Kg}$ of cordycepin in DMSO$\mathrm{NaCl} 0.1 \%$ solution or an IP injection of the vehicle $(\mathrm{NaCl} 0.1 \%) 5 x$ per week for 4 weeks. After the final treatment week, mice were sacrificed for molecular analysis.

\section{Human post-mortem brain tissue}

Post-mortem striatum and cerebellum brain tissue from two clinically and genetically SCA2 confirmed patients were obtained from the NIH NeuroBioBank (USA). Control striatum and cerebellum tissues from healthy individuals, without neurological conditions diagnosed, were obtained from NIH NeuroBioBank (USA). Tissues preserved in 4\% PFA solution, were dehydrated in a $30 \%$ sucrose/PBS for $48 \mathrm{~h}$, cryoprotected at $-80^{\circ} \mathrm{C}$ degrees, dissected in $40 \mu \mathrm{m}$ slices using a cryostat (Cryostar NX50, ThermoFisher Scientific) and stored in free floating PBS/sodium azide solution at $4^{\circ} \mathrm{C}$. 


\section{Immunochemical procedures}

Immunocytochemistry. Immunocytochemistry procedure was performed based on protocols described elsewhere [22, 23]. Briefly, for cells harvest, we washed cultures with PBS and fixed it with 4\% PFA for $20 \mathrm{~min}$. Cells were permeabilized with $1 \%$ Triton $^{\text {TM }}$ X-100 (Sigma) and blocked using 3\% Bovine Serum Albumin (Nzytech) in PBS. Incubation with primary antibodies was done overnight at $4{ }^{\circ} \mathrm{C}$. After PBS washes for antibodies removal, cells were incubated in secondary antibodies for $2 \mathrm{~h}$ at room temperature (RT). Finally, cells were washed with PBS and coverslips were mounted on microscope slides using Fluoromount- $\mathrm{G}^{\mathrm{TM}}$ medium with DAPI (4',6'-Diamidino-2'-phenylindole) (ThermoFisher Scientific). Images were acquired with 40x objective in a Andor benchtop spinning disk confocal instrument (Oxford Instruments, UK) and with a 63x and 100x objectives in a Zeiss LSM710 confocal microscope.

Immunohistochemistry - free floating incubation. Immunohistochemistry assays were performed based on protocols described elsewhere $[14,24,25]$. For visible microscopy analysis, mouse and human brain slices were incubated in PBS $/ 0.1 \%$ diphenylhydrazine for $30 \mathrm{~min}$ at $37^{\circ} \mathrm{C}$. A subsequent step of incubation in Tris-buffered $\mathrm{pH}=9$ antigen retrieval solution for $30 \mathrm{~min}$ at $95^{\circ} \mathrm{C}$ was performed for human sections. Then, all sections were left in PBS/10\% normal goat serum (Gibco)/0.1\% Triton X blocking solution for $1 \mathrm{~h}$ at RT, followed by overnight incubation with primary antibodies at $4{ }^{\circ} \mathrm{C}$. Posteriorly, slices were incubated in biotinylated secondary antibodies (1:200, Vector Laboratories) for $2 \mathrm{~h}$ at RT and then in a reaction with the Vectastain elite avidin-biotin-peroxidase kit and by 3,3'diaminobenzidine substrate (both from Vector Laboratories). After, sections were assembled over microscope slides, dehydrated in increasing degree ethanol solutions (75, 96 and $100 \%$ ) and xylene, and finally coverslipped using mounting medium Eukitt (O. Kindler $\mathrm{GmbH} \& \mathrm{CO}$, Freiburg, Germany). For fluorescence-labeling procedures, sections were incubated in the same blocking solution, followed primary and secondary antibodies incubation as described above. After antibodies probing, slices were mounted in microscope slides and coverslipped using the Fluoromount-G ${ }^{T M}$ medium with DAPI. Images were acquired with $20 x$ objective in a Zeiss Axio Imager Z2 and Axio Scan.Z1 Slide Scanner microscopes.

Immunochemical antibodies. For immunochemical procedures, the following primary antibodies were used: mouse anti-ataxin-2 (1:1000, ref. 611378, BD Biosciences); rabbit anti-DARP-32 (1:1000, ref. AB10518, Merck Millipore); mouse anti-lba1/AIF1 (1:1000, ref. MABN92, Merck Millipore); mouse anti-GFAP (1:1000, ref. 644702, BioLegend); rabbit anti-LC3B (1:1000, ref. NB100-2220, Novus); rabbit anti-SQSTM1 (1:1000, ref. 5114S, Cell Signaling); rabbit anti-Cleaved Caspase-3 (Asp175) (1:1000, ref. 9661, Cell Signaling); rabbit anti-Cleaved Caspase-3 (Asp175) (1:1000, ref. 9661, Cell Signaling); rabbit anti-GFAP (1:500, ref. Z0334, Dako, Agilent); rabbit anti-lba1 (1:1000, ref. 019-19741, FUJIFILM Wako Pure Chemical Corporation) and rabbit anti-NeuN (1:1000, ref. ABN78, Millipore).

\section{Cresyl violet staining}

Coronal mouse brain slices were displayed onto microscope slides, followed by cresyl violet solution staining and ethanol (75, 96 and $100 \%$ solutions) and xylene dehydration. Eukitt mounting medium was then used to mount coverslips. Images were acquired with $20 x$ objective in a Zeiss Axio Imager Z2.

Immunochemistry quantitative analysis. For quantification of ataxin-2 aggregates and DARPP-32 staining loss, 18 coronal sections per animal were analyzed in ZEN lite software (Zeiss), so that a complete rostrocaudal picture of the striatum was obtained (14-16 transduced sections). Ataxin-2 inclusions were manually counted in all animals in a blind manner. DARPP32 neuronal lesion area was manually measured for all animals, allowing quantification of depleted volume according to the formula: volume $=\mathrm{d}^{*}$ $(a 1+a 2+a 3)$, where $d$ is the distance between serial sections $(200 \mu \mathrm{m})$ and $\mathrm{a} 1+\mathrm{a} 2+\mathrm{a} 3$ are depleted areas for each individual section.

Cleaved caspase-3 quantitative analysis. The cleaved caspase-3 puncta were assessed by scanning 14-16 sections of each animal covering the entire transduced striatum using a 20x objective in an Axio Scan.Z1 Slide Scanner microscope. The total number of cleaved caspase-3 puncta was calculated automatically in a blind manner using Image $(\mathrm{NIH}, \mathrm{USA})$, as well as the number of ataxin-2 transduced cells in those sections [23]. Data were expressed as the ratio of the total number of cleaved caspase-3 puncta to the total number of ataxin-2 transduced cells (cordycepin versus control group), or as the ratio of the total number of cleaved caspase-3 puncta to the total number of ataxin-2 transduced cells normalized for the control Atx2WT hemisphere (lentiviral model).

Neuronal density quantitative analysis. The total number of ataxin-2 transduced cells was assessed by scanning 14-16 sections of each animal covering the entire transduced striatum using a 20x objective in an Axio Scan.Z1 Slide Scanner microscope. This number was calculated automatically in a blind manner using ImageJ (NIH, USA). Considering the striatal hemisphere volume of $10.75 \mathrm{~mm}^{3}$ (ref. [26]) and an average of $20 \%$ transduction area per hemisphere, the total number of ataxin- 2 transduced cells in the striatum was used to calculate the number of neurons transduced with ataxin- 2 per $\mathrm{mm}^{3}$.

\section{Western blot}

Cell extracts were lysed in 10x RIPA solution (Merck Millipore) and or mouse striatal punches were homogenized in a urea/DTT solution, both containing a cocktail of protease inhibitors (Roche pharmaceuticals), followed ultrasound sonication. Protein concentration levels were determined using Pierce ${ }^{\mathrm{T} M}$ BCA Protein Assay Kit (Thermo Scientific) for cell lysates and using NZYBradford reagent (Nzytech) for mouse samples. Sixty to eighty protein $\mu \mathrm{g}$ were resolved in SDS-polyacrylamide gels $(7.5 \%$ and $12 \%)$. Followed by protein transfer to PVDF membrane (Merck Millipore) and antibody probing using the following antibodies: mouse anti-ataxin-2 (1:1000, ref. 611378, BD Biosciences); mouse anti-IC2 (polyQ chain) (1:1000, ref. MAB1574, Merck Millipore); rabbit anti-LC3B (1:1000, ref. NB100-2220, Novus); rabbit anti-SQSTM1 (1:1000, ref. 5114S, Cell Signaling); mouse anti$\beta$-actin (1:5000, ref. A5316, Sigma Aldrich); rabbit anti-DARP-32 (1:1000, ref. AB10518, Merck Millipore). Optical densiometric analysis was carried out using Image J software (USA).

\section{Quantitative real-time PCR}

Total RNA from mouse striatal punches was extracted with NZY Total RNA Isolation kit (Nzytech), after a first step of Trizol (Invitrogen) tissue dissociation and RNA/DNA/protein chloroform separation. RNA concentration and purity were assessed using the NanoDropTM 2000 (Thermo Scientific). CDNA molecules of $1 \mu \mathrm{g}$ of RNA were synthesized according to iScript cDNA kit (Bio-Rad) in a 96-well thermocycle (Bio-Rad). For quantitative RT-PCR, it was used SsoAdvanced ${ }^{\mathrm{TM}}$ Universal SYBR ${ }^{\circledast}$ Green Supermix (Bio-Rad) in a CFX96 Touch Real-Time PCR Detection System (BioRad). The Mouse Autophagy Primer Library (MATPL-1, Biomol), containing primers for 88 genes related to autophagy and for 8 housekeeping genes, and accordingly RT-PCR conditions were used. mRNA expression levels relative to mRNA gene control were determined using amplification values.

\section{Statistical analysis}

All statistical analysis was performed using Student's t-test or one-way ANOVA followed by post hoc Tukey's multiple comparison test using GraphPad software (La Jolla, USA). Results are normally expressed as mean \pm SEM. Significant thresholds were set at $P<0.05, P<0.01, P<0.001$ and $P$ $<0.0001$, as defined in the text.

\section{RESULTS}

Overexpression of mutant ataxin-2 leads to neuropathological and behavior abnormalities SCA2-related

The development and characterization of adequate non-human models of disease is crucial to elucidate disease mechanisms and to investigate new therapies. For SCA2, different cellular and animal models have been developed [27]. While the use of these models has several advantages, often they only display disease features in advanced ages, which sometimes conditionate the development of research projects in a reasonable timeline. The classical view is that SCA2 selectively impacts the cerebellum, leading to poor movement coordination as the disease progresses. However, clinical evidence from the past 10 years shows that other regions are affected and are the underlying causes for non-ataxia symptoms [6]. Therefore, we aimed to develop a new model based in the expression of mutant ataxin-2 through LVs in the striatum region. A similar approach has been used for 

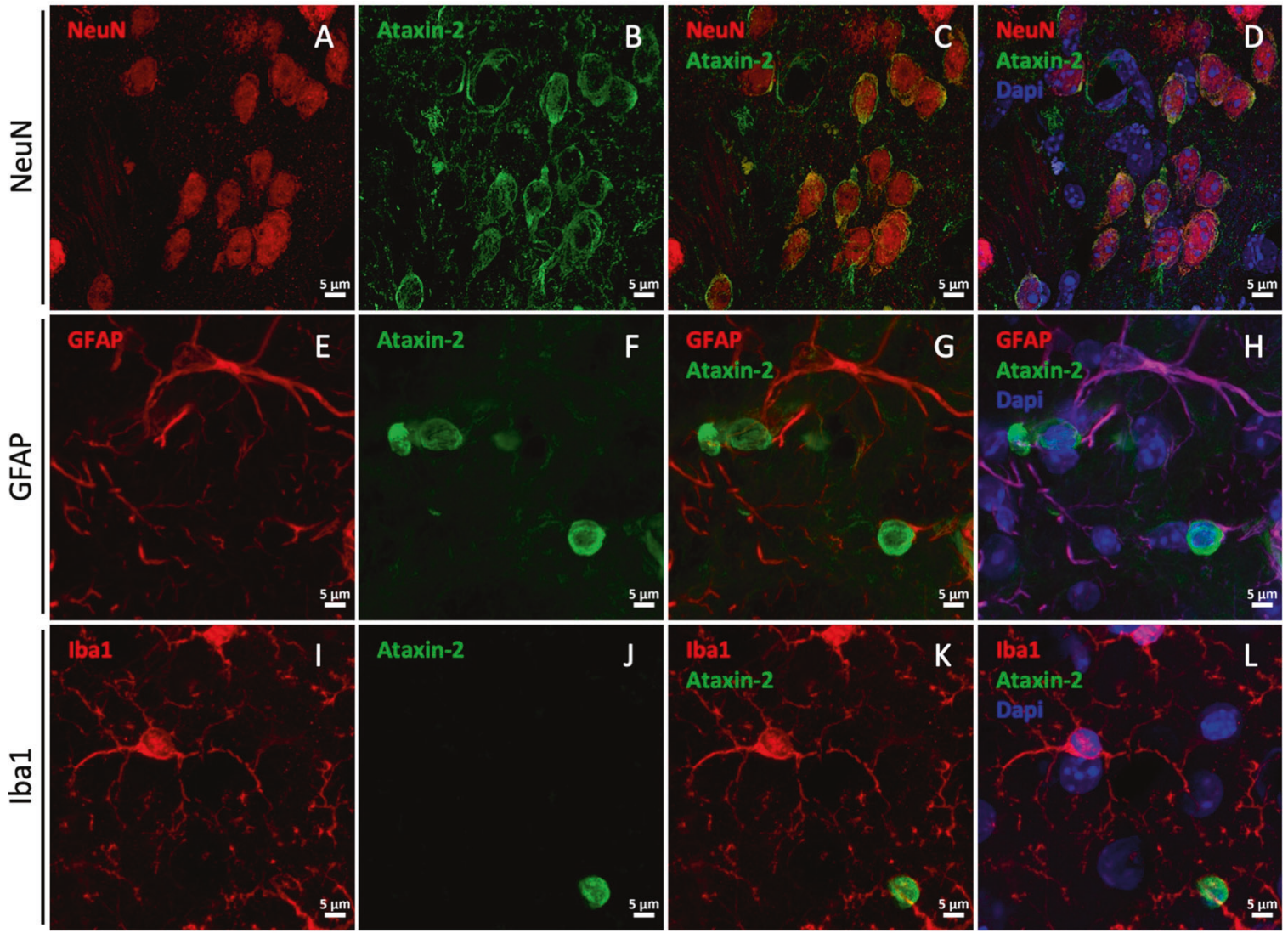

Fig. 1 Lentiviral vectors (LVs) encoding for ataxin-2 specifically transduces striatal neurons. LVs encoding for human wild-type ataxin-2 (Atx2WT) and mutant ataxin-2 (Atx2MUT) were injected in mice striatum under the control of neuron-specific PGK promoter. Brain sections were immunoassayed for ataxin-2 protein, the nuclear neuronal marker NeuN, and the glial markers GFAP and Iba1. A-D Confocal microscopy revealed that ataxin-2 is expressed in NeuN positive neurons, surrounding the nucleus of these cells. On the other hand, GFAP marker and Iba1 co-staining showed that LVs do not transduce glial cells, as ataxin-2 expression does not co-localized with astrocytes (E-H) or microglia (I-L).

Huntington's disease and MJD (ref. [28, 29]). Previously, it was shown that LVs have a high tropism to transduce neurons [19]. Accordingly, we showed that LVs promote the expression of ataxin-2 in striatal neurons, stained for the neuronal nuclear marker NeuN, regardless of the length of the polyglutamine expansion (Fig. 1A-D). Moreover, LVs did not transduce glial cells, such as astrocytes or microglia, as co-localization between ataxin2 and the glial markers GFAP and Iba1 was not observed (Fig. $1 \mathrm{E}-\mathrm{L})$. Additionally, ataxin-2 expression was under the control of the mouse phosphoglycerate kinase 1 (PGK) promoter, which allows long-term and sustained transduction of striatum neurons $[24,29]$. To develop the striatal lentiviral model for SCA2, LVs encoding for human wild-type ataxin-2 (Atx2WT) were injected in mice left striatum, while mutant ataxin-2-encoding vectors (Atx2MUT) were injected in the contralateral hemisphere of the same animal, through stereotaxic surgery (Fig. 2A). These constructs were previously validated in N2A cells, in which we observed the presence of ataxin-2 aggregates upon transfection with Atx2MUT (Supplementary fig. 1). First, we analyzed a group of injected animals to confirm the expression of ataxin- 2 in both hemispheres through western blot analysis of striatal punches (Supplementary fig. 2A, B). No differences were found in the expression of Atx2WT and Atx2MUT. For immunohistochemical analysis, the injected animals were divided into three different groups and sacrificed at 4-, 8- and 12-weeks post injection (Fig. $2 B)$. In these animals, we quantified the number of transduced neurons in the striatum per $\mathrm{mm}^{3}$, by analyzing the transduced sections and automatically counting the ataxin- 2 positive cells. In the same line as what was observed in the western blot analysis, no differences in the expression between Atx2WT $(20,609 \pm 2091)$ and Atx2MUT $(22,656 \pm 1,792)$ was observed, thus suggesting similar levels of LVs transduction (Supplementary fig. 2C-G). Mutant ataxin-2 protein is prone to aggregate and form cytoplasmic insoluble inclusions that are found in patients' brain tissue [30]. Immunohistochemistry analysis for ataxin-2 revealed a diffuse staining across Atx2WT injected region (Fig. 2C), while we detected the presence of ataxin-2 aggregates in the Atx2MUT injected hemisphere (Fig. 2D-F). The number of aggregates increased significantly over time, reaching the maximum at 12 weeks post injection (Fig. 2K). We also confirmed, through confocal microscopy, that ataxin-2 aggregates are located in cells cytoplasm surrounding the nucleus, in line to what was reported in previous studies [31] (Supplementary fig. 3). In the MJD lentiviral model, the expression of mutant ataxin-3 resulted in the loss of neuronal markers [29]. In line with this, we found that the expression of Atx2MUT led to a depletion of DARPP-32 marker, being the volume of neuronal loss significantly higher at 12 weeks post injection compared to the 4- and 8- weeks timepoints (Fig. $2 \mathrm{H}-\mathrm{J}, \mathrm{L})$. In the Atx2WT hemisphere there is a residual DARPP-32 staining depletion (Fig. 2G), although it was lower than the marker loss reported upon an injection with PBS (ref. [32]). Importantly, the levels of DARPP-32 loss were similar across all time points (Supplementary fig. 4). These results show that the expression of mutant ataxin-2, induces neuropathological alterations similar to those observed in SCA2 patients [33, 34].

Neuronal degeneration in different brain regions is typically correlated with behavioral manifestations. Particularly, several studies have shown that striatal dysfunction could lead to a wide 
A

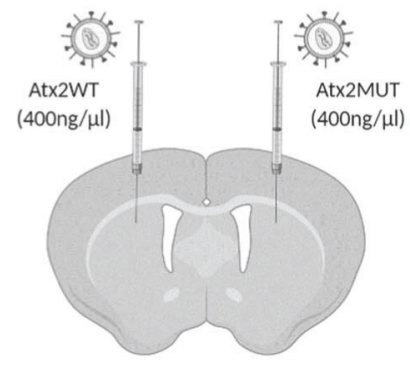

B

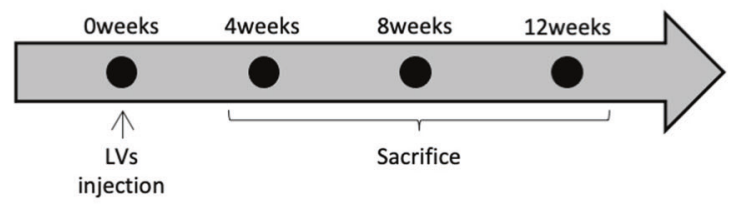

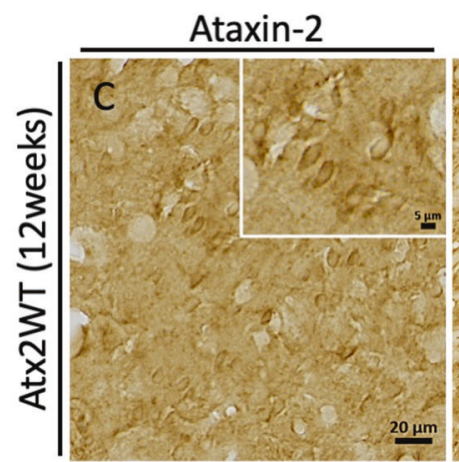
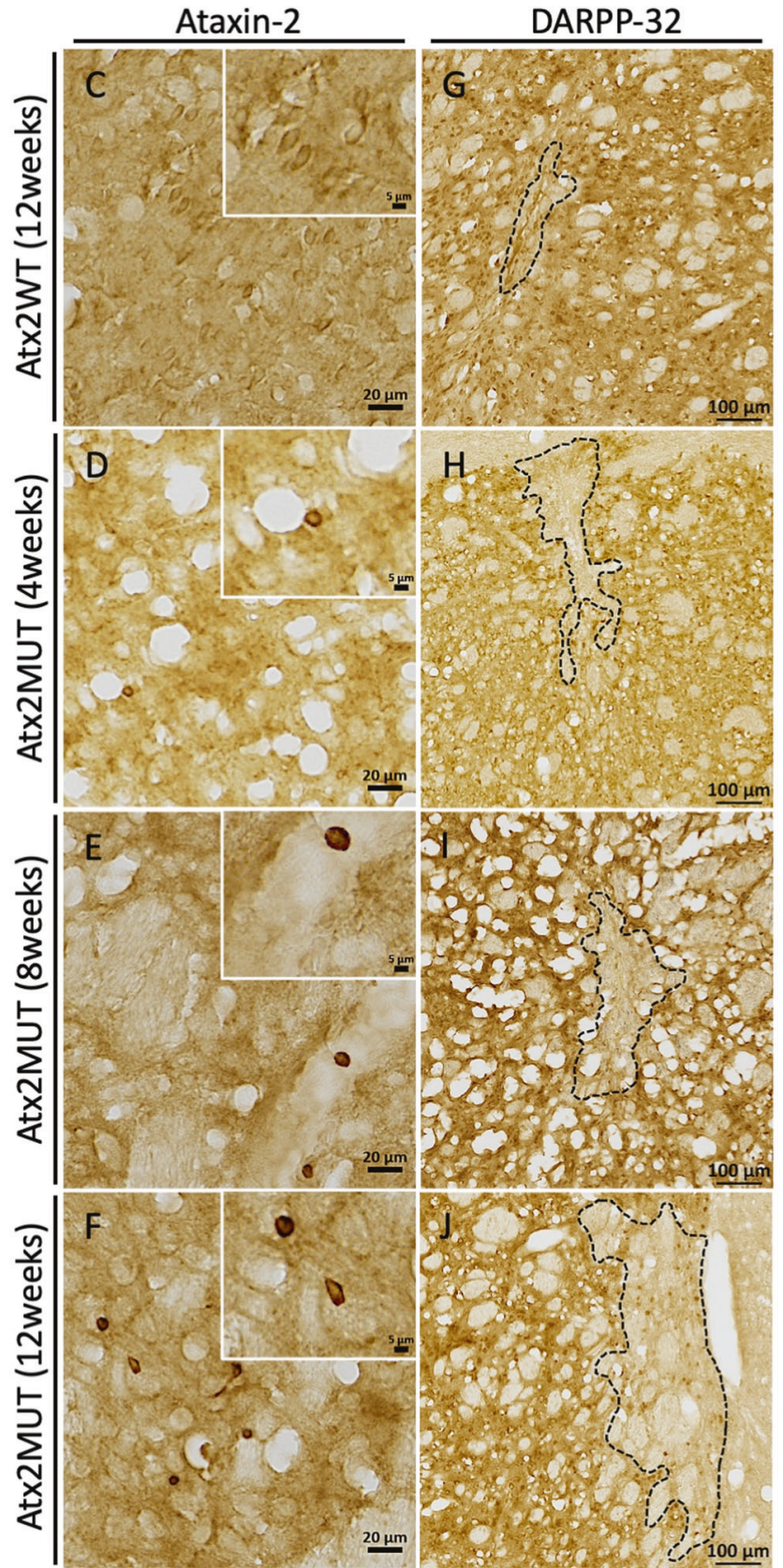

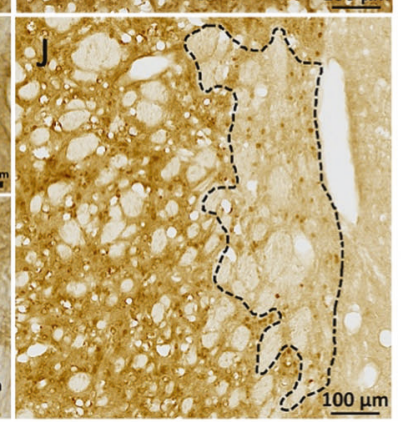

K
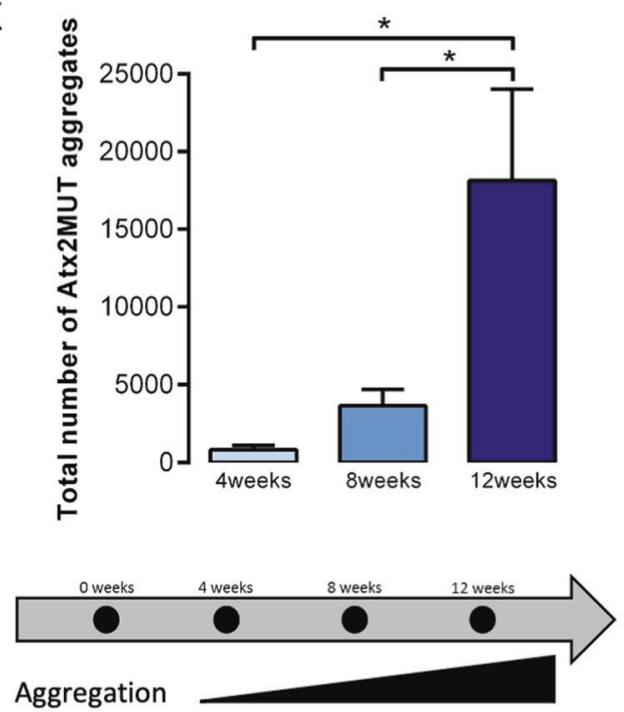

$\mathrm{L}$
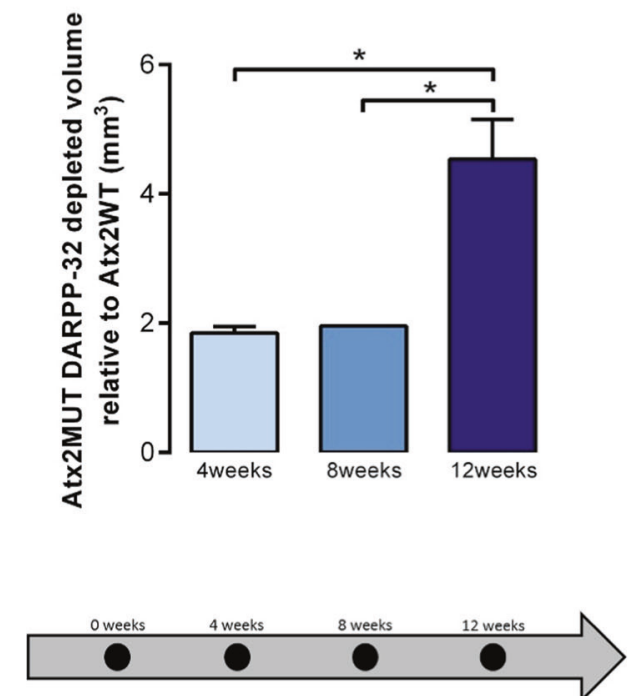

Neuronal lesion range of cognitive and behavioral signs including affective and motivational disturbances, impaired reward processing and anxiety [35]. For that, we injected animals unilaterally with either Atx2WT or Atx2MUT and subjected them to open field test, comparing them also to WT animals. At four weeks post injection, all groups showed similar results in distances covered, zones explored and rearing behavior (Supplementary fig. 5). However, at eight weeks post injection we observed a hyperactive behavior in the Atx2MUT-injected animals compared to the animals injected with Atx2WT or with non-injected animals (Supplementary fig. 6). 
Fig. 2 The expression of mutant ataxin-2 in the striatum induces aggregation and loss of neuronal marker. A Animals received a stereotaxic injection with lentiviral vectors (LVs) encoding for human wild-type ataxin-2 (Atx2WT) and mutant ataxin-2 (Atx2MUT) in left and right striatum hemisphere, respectively. B Animals were divided in three groups according to the time of ataxin-2 expression, for 4-, 8- and 12weeks after injection. C Immunohistochemistry of brain sections revealed a general diffuse signal for ataxin-2 protein expression in Atx2WT hemisphere. In contrast, the overexpression of Atx2MUT resulted in ataxin-2 aggregates at 4 (D), 8 (E), and 12 (F) weeks of experiment. $\mathbf{K}$ The quantification of the total number of mutant aggregates showed a significant increase over time of ataxin-2 expression, reaching high levels at 12 weeks (values are expressed as mean \pm SEM $n=3$ per timepoint; ${ }^{*} P<0.05$; one-way ANOVA followed by post hoc Tukey's multiple comparisons test). G Immunohistochemistry for DARPP-32 neuronal marker showed that Atx2WT results in reduced marker loss, whereas Atx2MUT induces a higher depletion of staining for DARPP-32 either at 4- and 8-weeks post injection $(\mathbf{H}, \mathbf{I})$. These alterations are particularly high at 12 weeks $(\mathbf{J}, \mathbf{L})$ (values are expressed as mean \pm SEM $n=3\left(4-, 8\right.$-weeks) and $n=8$ (12-weeks); ${ }^{*} P<0.05$; one-way ANOVA followed by post hoc Tukey's multiple comparison test).

Specifically, mice expressing expanded ataxin-2 explored longer distances and performed more rearing compared to control groups, which could be suggestive of a hyperactive exploratory behavior [36].

Altogether, these results show that the expression of mutant ataxin- 2 in the striatum leads to the development of behavior and neuropathological abnormalities SCA2-related.

\section{The expression of mutant ataxin-2 leads to neuroinflammation and cell death}

Neuronal death is the ultimate outcome of the molecular events underlying the SCA2 neuropathological process, which might be related to apoptosis. Therefore, in the injected animals we analyzed cleaved caspase-3 labeling (Fig. 3A-D), the active form of caspase-3 protein, which plays a critical role in apoptosis [37]. We observed that the expression of Atx2MUT led to a significant increase in cleaved caspase-3 puncta over the different timepoints of the experiment, suggesting an increase in cell death through apoptosis (Fig. 3Q). Moreover, the formation of pycnotic nuclei structures occurs during the apoptotic process, as chromatin becomes condensed [38]. To assess the presence of these structures upon Atx2MUT expression, we performed a cresyl violet staining of striatal sections. In line with the results depicted above, we observed an increased presence of pycnotic nuclei over time of Atx2MUT expression, suggesting possible cell injury and striatal degeneration (Fig. 3E-H).

Neuroinflammation has been reported in polyQ diseases, as well as in other neurodegenerative disorders $[39,40]$. In SCA2, reactive astrocytes and microglia, characterized by the expression of GFAP and Iba1 markers, respectively, were observed in different brain tissues of affected individuals $[2,41,42]$. In line with this, we found that Atx2MUT resulted in marked astrogliosis (Fig. $3 \mathrm{~J}-\mathrm{L}$ ) and microgliosis (Fig. $3 \mathrm{~N}-\mathrm{P}$ ) across mice striata, compared to a minimal presence of reactive astrocytes and microglia in the needle insertion site in the Atx2WT injected hemisphere (Fig. 3I, M). The triggering of neuroinflammation by Atx2MUT is highlighted by the fact that surgically associated gliosis around the injection site is typically reduced over time, while we found that inflammatory response markers reactivity in Atx2MUT injected hemisphere increased over time (Fig. 3M). Overall, these results indicate that expression of mutant ataxin-2 leads to cell injury and degeneration in mice striatum, while also inducing neuroinflammation.

\section{Autophagy pathway is impaired in SCA2 models}

Several studies have already shown the involvement of autophagy in polyQ pathologies [13], however evident dysfunction of this pathway in SCA2 is still unclear. Thus, we aimed at studying autophagic markers in several SCA2 models. First, we used Neuro-2A cells transfected with different constructs of human ataxin-2, carrying different number of polyQ repeats: Atx2Q22 (WT form), Atx2Q58 and Atx2Q104 (MUT forms) (ref. [20]). We then analyzed the protein levels of SQSTM1, which recognizes ubiquitinated molecules and direct them to autophagosomes [43], and LC3B, responsible for autophagosome maturation [44] (Fig. 4A). We found increased levels of SQSTM1 when cells were expressing mutant ataxin-2 compared to control condition (Fig. 4B). Regarding LC3B protein, we observed a significant decrease in the LC3B-II levels when cells were expressing mutant ataxin-2 forms compared to wild-type ataxin-2 transfected and non-transfected cells (Fig. 4C). These alterations suggest a dysregulation of autophagy pathway upon mutant ataxin-2 expression. Importantly, the levels of these two autophagy markers were similar between non transfected cells and cells expressing the wildtype ataxin- 2 protein.

We then investigated the protein levels of SQSTM1 in striatum samples of the lentiviral mouse model (Fig. 4D). In agreement with the in vitro results, we found significantly increased levels of SQSTM1 marker in the hemisphere expressing mutant ataxin-2 compared to control hemisphere (Fig. 4E, F). Additionally, we performed an mRNA array comprising different genes related to the autophagy process. In line with the previous results, we found elevated SQSTM1 gene expression levels (Fig. 4G), as well as BCl2 gene (Fig. $4 \mathrm{H}$ ) in the hemisphere expressing mutant ataxin-2, compared to the samples expressing the wild-type ataxin-2 protein. Altogether, these data suggest that expression of mutant ataxin-2 leads to abnormal alterations in the autophagy pathway.

\section{Abnormal accumulation of autophagic markers is detected in SCA2 patients' brain tissue}

Several studies have already demonstrated accumulation of autophagic vesicles and markers in neuronal tissues of individuals suffering from polyQ [14]. So far, besides the analysis of SCA2 patients peripheral blood cells [17], no evidences of autophagic dysfunction were described in SCA2 patients' brain. Upon microscopy analysis, we confirmed the presence of the hallmark mutant ataxin-2 aggregates in the cerebellum of SCA2 patients (Fig.5D), while these aggregates were not observed in healthy individual brain samples (Fig. 5D). Importantly, we detected an abnormal accumulation of LC3B (Fig. 5E) and SQSTM1 (Fig. 5F) markers in SCA2 patients' brain tissues, comparing to the expression observed in non-disease control neuronal tissue (Fig. 5B, C). Furthermore, this abnormal accumulation of autophagy markers seems also to be observed in striatum tissue from SCA2 patients, compared to healthy individuals (Supplementary fig. 7). These important pathological findings show to our knowledge the first evidence of an autophagic impairment in the brain of SCA2 patients.

\section{Pharmacologic activation of autophagy through cordycepin} mitigates SCA2-associated abnormalities

As post-mitotic cells, neurons rely on efficient degradation systems to maintain protein homeostasis [45]. It was shown that dysfunction of these systems, particularly autophagy, contribute to the pathogenesis of neurodegenerative disorders [15]. In line with these studies, we show that the pharmacological inhibition of autophagy results in an increase of the accumulation of aggregates of either wild-type or mutant ataxin-2 (Supplementary fig. 8). Thus, we next aimed to rescue neuropathological abnormalities associated with SCA2, by molecularly inducing autophagy, using the bioactive compound cordycepin or $3^{\prime}$-deoxyadenosine. We have previously shown that cordycepin induces activation of adenosine monophosphateactivated protein kinase (AMPK) pathway, impacting the autophagy 

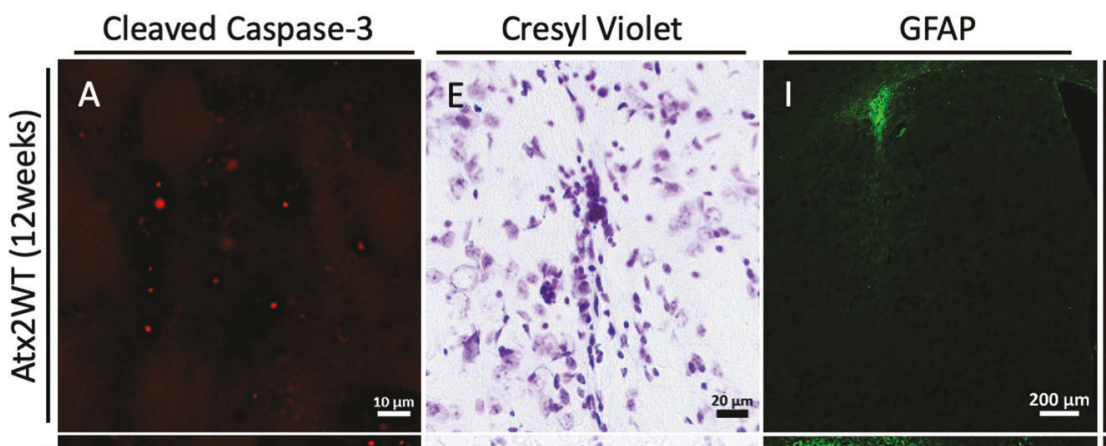

Iba1
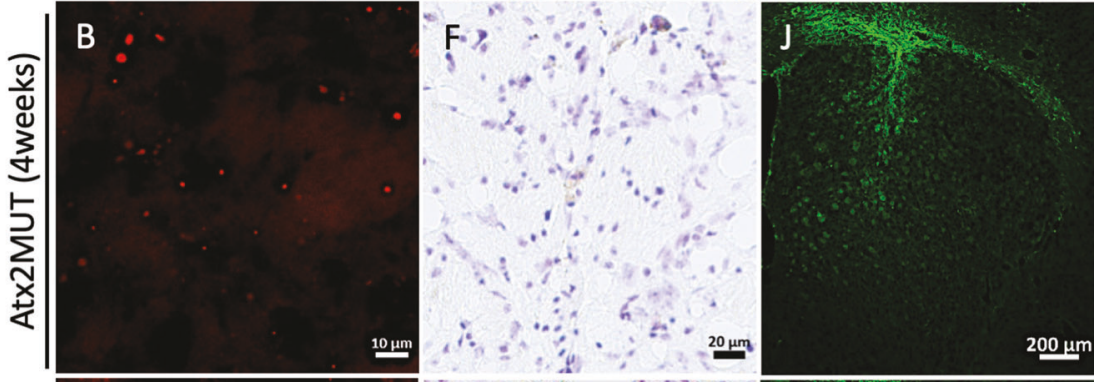

\section{M}
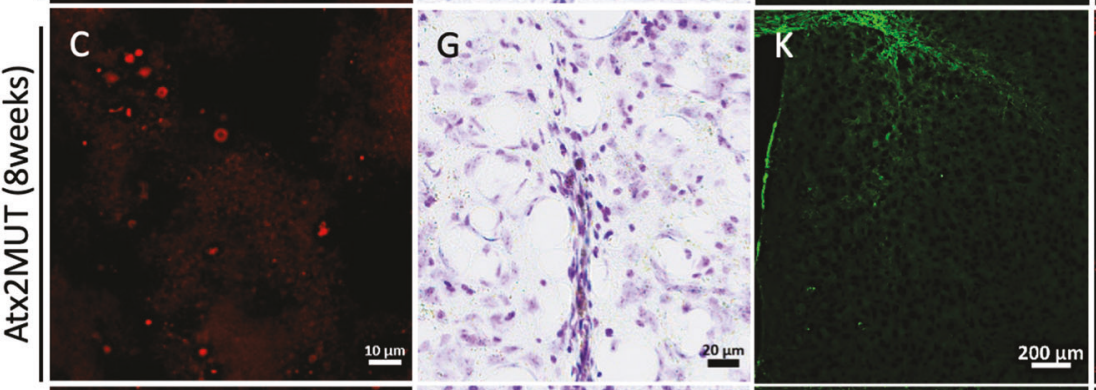

0

N
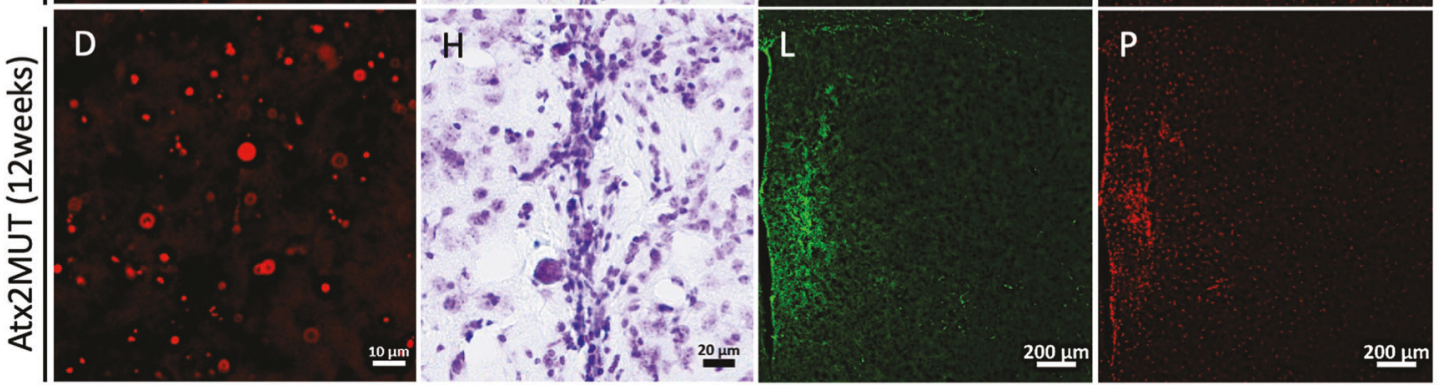

Q

$\mathrm{R}$
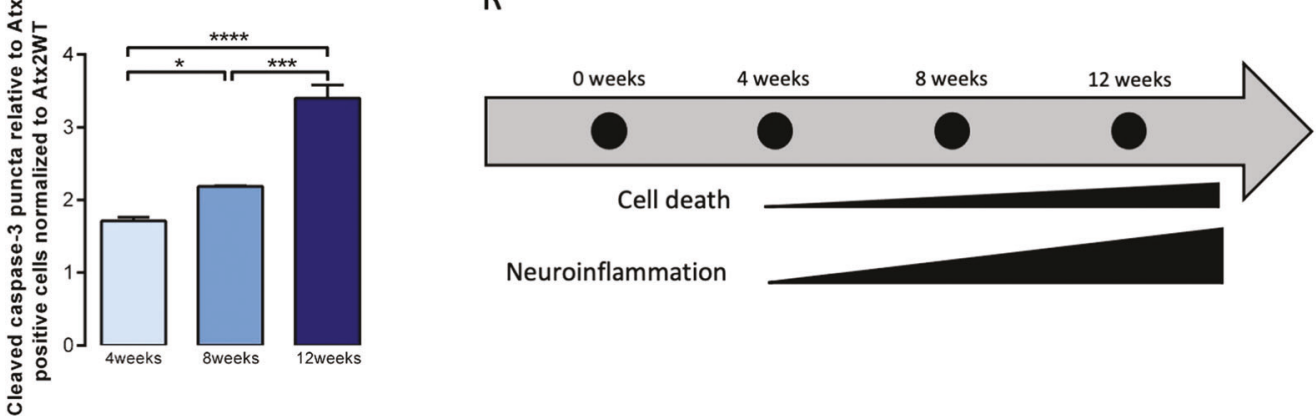

Fig. 3 The expression of mutant ataxin-2 leads to cell death and neuroinflammation. Cleaved caspase- 3 immunostaining of mouse striatum sections injected with Atx2WT at 12 weeks (A) and Atx2MUT at 4-, 8- and 12- weeks postinjection (B-D). Quantification of cleaved caspase-3 puncta relative to Atx2 positive cells showed that expression of Atx2MUT in the mouse striatum leads to a significant increase of cleaved caspase-3 over the different timepoints, suggesting an increase in apoptosis (Q) (values are expressed as mean \pm SEM $n=3$ per timepoint and normalized to Atx2WT; ${ }^{*} P<0.05$, ${ }^{* * *} P<0.001,{ }^{* * * *} P<0.0001$; one-way ANOVA followed by post hoc Tukey's multiple comparison test). E-H Cresyl violet staining revealed that expression of Atx2MUT resulted in increased presence of pycnotic nuclei structures over time, suggesting cell lesion and striatal degeneration (R). Immunohistochemistry for GFAP (astrocytes marker) and Iba-1 (microglia marker) revealed low levels of astrogliosis (I) and microgliosis (M) upon expression of Atx2WT. In contrast, the overexpression of Atx2MUT induced an inflammatory response, highlighted by increased staining of GFAP (J-L) and Iba-1 (N-P) at 4-, 8- and 12-weeks after disease beginning. These neuropathological alterations are higher at 12-weeks of experiment (R). 
A

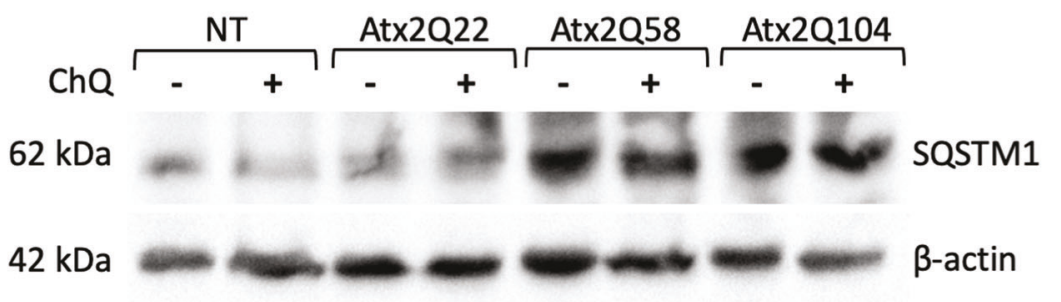

$17 \mathrm{kDa}=\ldots+\ldots$ LC3B-I

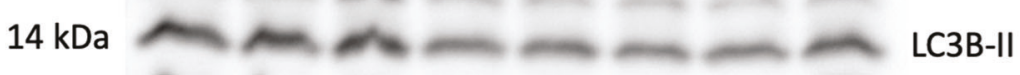

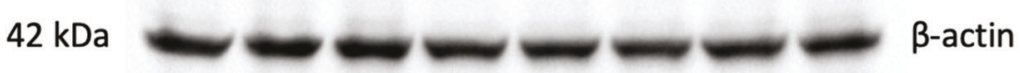

B

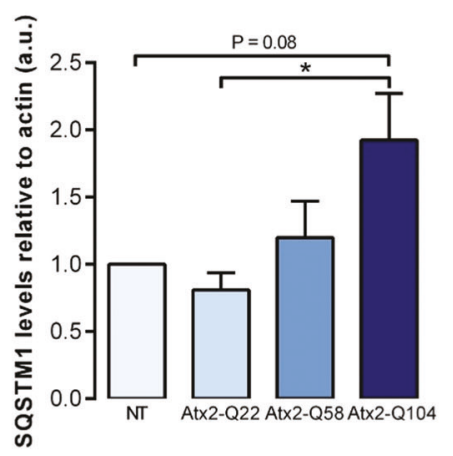

D

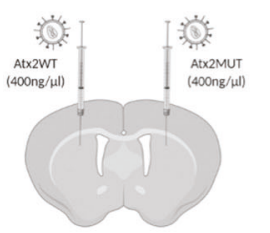

G
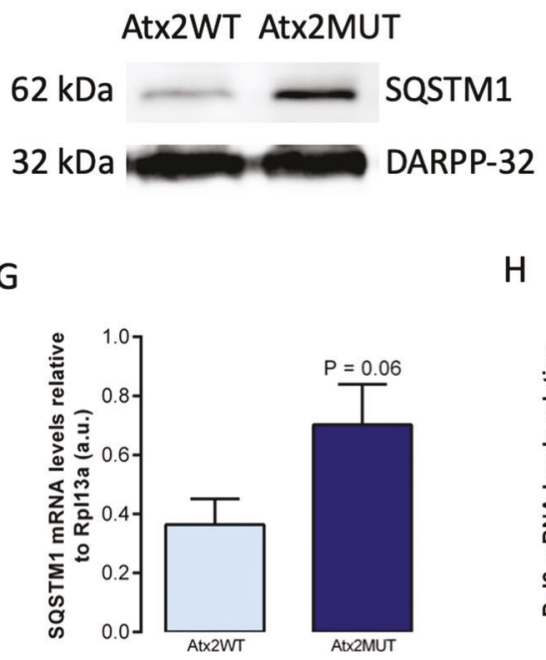

C

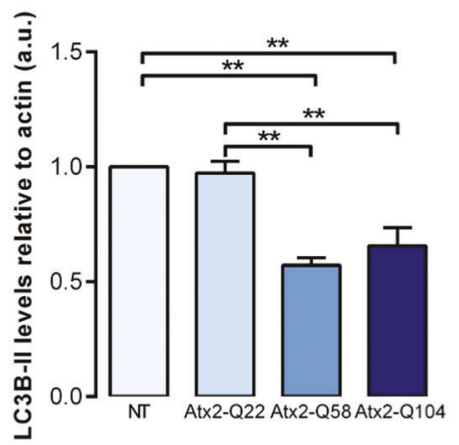

$\mathrm{F}$

Fig. 4 Autophagic markers levels are altered in SCA2 models. Cultured cells from mouse neuroblastoma cell line (Neuro-2A) were transfected either with human wild-type ataxin-2 or mutant ataxin-2 forms, and cells extracts were collected $48 \mathrm{~h}$ after transfection. A Representative western blot analysis of autophagic markers, revealed increased levels of SQSTM1 (B) and decreased levels of LC3B-II (C) upon expression of mutant ataxin-2, compared to non-transfected (NT) and wild-type ataxin-2 conditions. Data are expressed relative to $\beta$-actin levels ( $n=3$ independent experiments). D Striatal punches of animals injected with Atx2WT and Atx2MUT were collected for protein and RNA extraction. E Western blot analysis showed significantly increased levels of SQSTM1 in the presence of Atx2MUT compared to Atx2WT mice (F). Results are expressed relative to DARPP-32 levels $(n=8)$. Quantitative RT-PCR reaction indicated upregulation of SQSTM1 $(\mathbf{G})$ and Bcl2 $(\mathbf{H})$ mRNA gene levels in Atx2MUT animals compared to control. Results are expressed relative to Rpl13a levels $(n=6)$. Values are expressed as mean \pm SEM; ${ }^{*} P<0.05,{ }^{* *} P<0.01$; unpaired Student's $t$-test; one-way ANOVA followed by post hoc Tukey's multiple comparison test).

process [16]. Moreover, we observed that cordycepin resulted in decreased levels of LC3B-II and SQSTM1, suggesting an increase of autophagic clearance. Importantly, cordycepin treatment led to mitigation of neuropathological and motor deficits observed in mouse models of MJD. In this study, using Neuro-2A cells we found that $20 \mu \mathrm{M}$ cordycepin treatment led to a significant reduction in the number of cells with mutant ataxin-2 aggregates (Supplementary fig. 9). Next, we went to analyze cordycepin impact in the SCA2 striatal lentiviral mouse model. Briefly, mice expressing Atx2MUT (Fig. 6A) were treated with intraperitoneal injections of $20 \mathrm{mg} / \mathrm{Kg}$ of 

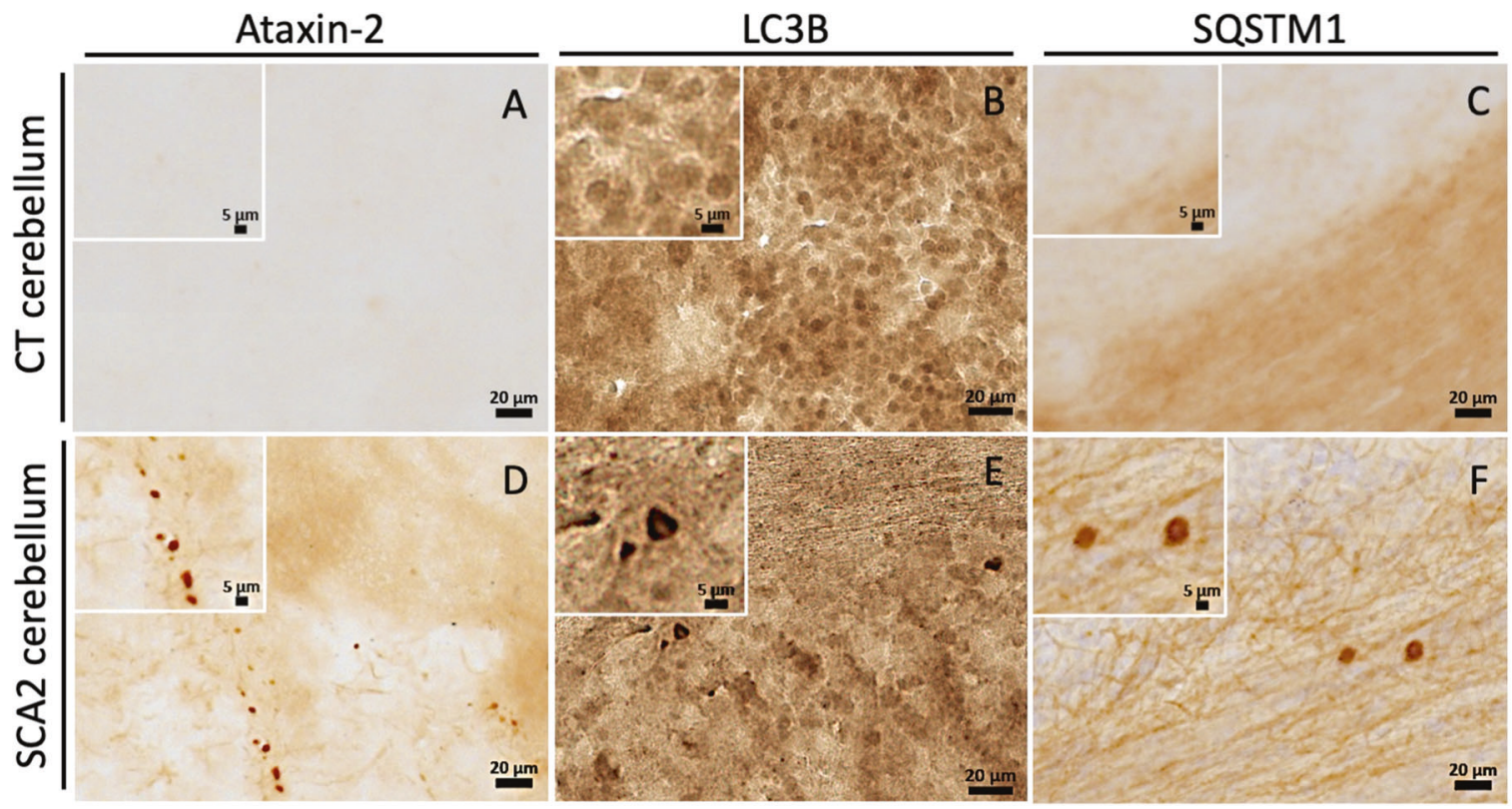

Fig. 5 Autophagy markers abnormally accumulate in SCA2 patients' brain. Immunohistochemical analysis of cerebellum post-mortem brain sections of SCA2 patients and healthy controls. A Staining for ataxin-2 showed no immunoreactivity in cerebellum tissue of healthy control. D On the other hand, staining for ataxin-2 revealed the presence of aggregated structures in cerebellum of SCA2 brain, indicating a disease state. Staining for LC3B and SQSTM1 autophagic markers demonstrated abnormal puncta accumulation of these proteins in SCA2 cerebellum (E, F), compared to healthy individual tissues $(\mathbf{B}, \mathbf{C})$.

cordycepin for 12 weeks, while control animals received the saline vehicle (Fig. 6B). We found that cordycepin administration resulted in a significant decrease in the number of ataxin-2 aggregates, compared to the control animals (Fig. 6C-E). Moreover, we performed a short-term treatment of four weeks with intraperitoneal injections of $20 \mathrm{mg} / \mathrm{Kg}$ of cordycepin or the saline vehicle being administered in mice expressing Atx2MUT to assess ataxin-2 protein levels (Supplementary fig. 10A). In line with the reduction of aggregates, we found a reduction of ataxin- 2 protein levels in the cordycepin-treated animals, compared to the control group (Supplementary fig. 10G). Also, we observed a significant preservation of DARPP-32 neuronal marker staining volume in the cordycepintreated animals (Fig. $6 \mathrm{~F}-\mathrm{H}$ ), compared to non-treated group. In the same line, we found that cordycepin treatment resulted in reduced cleaved caspase- 3 puncta relative to ataxin- 2 positive cells, compared to saline vehicle treated animals (Fig. 6I-K). In addition, we assessed whether cordycepin treatment could impact the neuroinflammation abnormalities of the SCA2 lentiviral mouse model. In fact, we observed lower levels of GFAP and lba1 immunoreactivity upon treatment with cordycepin, compared to control animals (Supplementary fig. 10C-G). To further demonstrate that cordycepin is inducing the autophagic activity, Neuro-2A cells were transfected with a plasmid expressing LC3B-GFP and -RFP puncta that refers to autophagosomes or autolysosomes, respectively (Supplementary fig. 11). Cells treated with $20 \mu \mathrm{M}$ cordycepin showed significant higher levels of LC3B-GFP puncta, like starvation condition, indicating increased levels of autophagosomes and suggesting activation of autophagy. Overall, our results support a beneficial role of cordycepin in SCA2 neuropathology, suggesting the upregulation of autophagy as a potential target to treat this pathology.

\section{DISCUSSION}

SCA2 is an incurable and fatal disease prompting devastating physical and psychological consequences for affected individuals and their families. The causative polyQ-expanded ataxin-2 protein is responsible for the cascade of events that precede neuronal death and degeneration of different brain regions [41]. However, the exact mechanisms underlying SCA2 pathogenesis are still controversial and need further elucidation so that a diseasemodifying therapy can be developed. Disease models are fundamental tools to study physiological pathways behind human pathologies. Basic models as yeast, Caenorhabditis elegans and Drosophila melanogaster allowed the study of ataxin-2 biological roles $[46,47]$. On the other hand, more complex models, like murine animals, permit a greater and profound functional knowledge, as well as the investigation of therapeutic solutions. The existing SCA2 mouse models have several advantages and have provided valuable insights in disease research [27, 48-53].

While the most affected brain region in SCA2 patients is the cerebellum, and specifically the Purkinje cells [31], it is now clear that several other regions are also profoundly affected in these patients and responsible for the non-ataxia symptoms that SCA2 patients also display [6]. However, there is a lack of studies focusing on the striatum or other regions affected in SCA2, despite the fact that mutant ataxin- 2 inclusions and neurodegeneration were observed in the striatum in patients' brain [31, 33, 34, 54]. In fact, the involvement of this region in the disease could explain the parkinsonism symptoms observed in several SCA2 patients [55]. Therefore, in this study we aimed at developing a mouse model featuring striatal pathology through the expression of mutant ataxin-2 under the control of neuron-specific PGK promoter mediated by lentiviral vectors, which are characterized by neuronal tropism and long-term stable expression [56].

Using this strategy, we detected mutant ataxin-2 aggregates and loss of a neuronal marker, as early as 4 weeks after the injection of LVs encoding mutant ataxin-2. These neuropathological alterations increased significantly over time and reached maximum at 12 weeks post injection. Such early SCA2 signs were only detected in the Q127 transgenic mice, in which perinuclear aggregates were detected at 4 weeks of disease, although no sights of neuronal loss was found [51]. Moreover, the expression of mutant ataxin-2 resulted in increased levels of cleaved caspase-3 puncta and appearance of condensed chromatin pycnotic nuclei, suggesting cellular injury and death. Further characterization of our model strengthened the role of glial cells in SCA2 
A

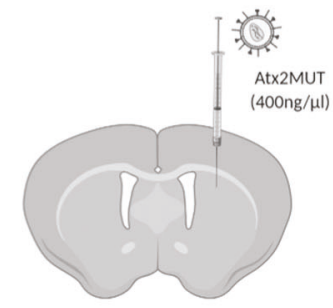

B

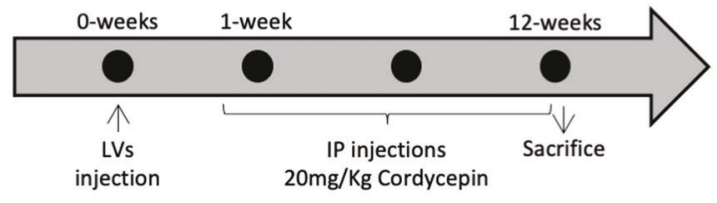

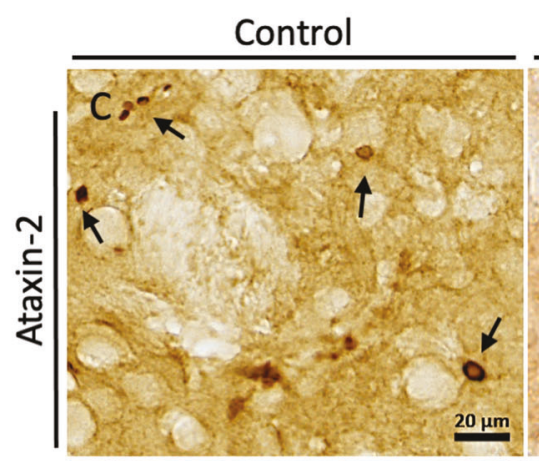
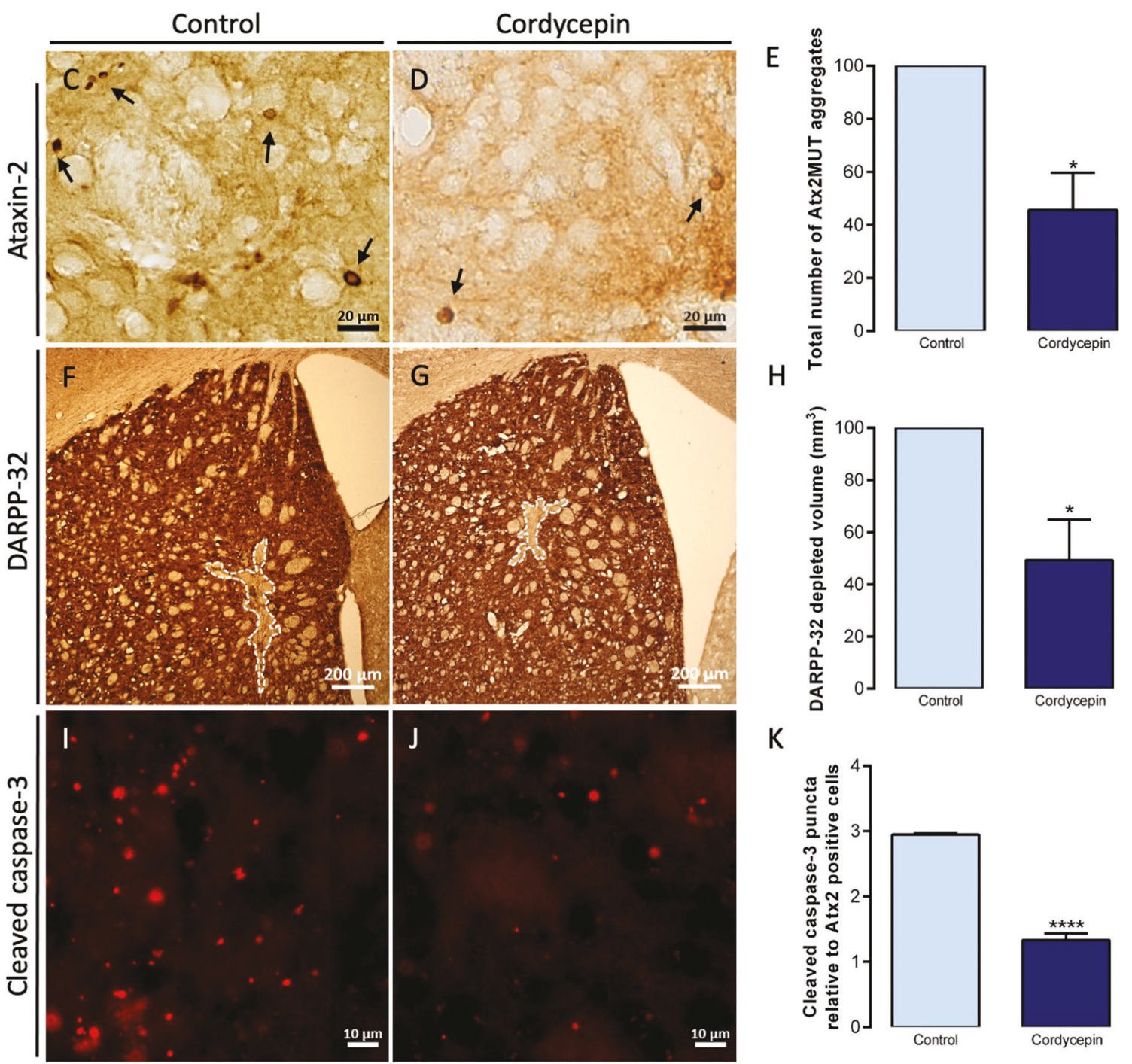

$\mathrm{H}$

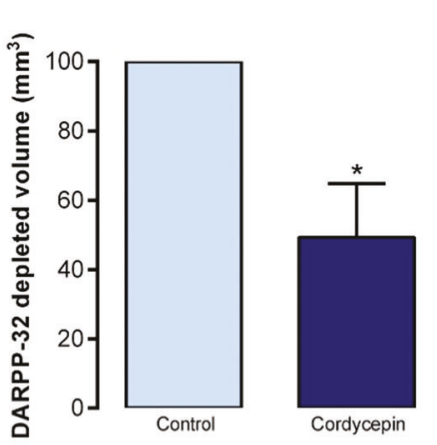

K

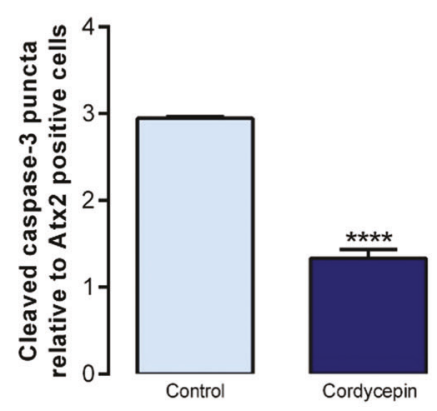

Fig. 6 Autophagy upregulation through cordycepin leads to reduced aggregation and neuronal marker loss in the SCA2 striatal mouse model. A, B Lentiviral mouse model, expressing Atx2MUT in the right striatum hemisphere, was intraperitoneally administered either with the vehicle ( $\mathrm{NaCl} 0.1 \%$ ) or $20 \mathrm{mg} / \mathrm{Kg}$ of cordycepin (in DMSO- $\mathrm{NaCl} 0.1 \%$ solution) for 12 weeks. C-E Immunohistochemistry of brain sections for ataxin-2 revealed a decrease in the total number of mutant ataxin-2 aggregates upon cordycepin treatment. F-H Immunohistochemistry staining of DARPP-32 neuronal marker showed a reduction in the depleted volume in treated animals compared to the control group. I-K Cordycepin treatment resulted in a significant decrease of cleaved caspase-3 puncta relative to ataxin-2 positive cells, compared to nontreated animals (values are expressed as mean \pm SEM $n=3$ (control) and $n=4$ (cordycepin) relative to control group mean; ${ }^{*} P<0.05$, ${ }^{*} P<$ 0.0001 ; unpaired Student's $t$-test).

pathogenesis. Neuroinflammation actively contributes to increased toxicity and neuronal death in many neurodegenerative disorders [40]. This process is mediated by astrocytes and microglia cells, that secrete neurotrophic factors and cytokines [57]. In fact, reactive astrocytes and microglia are present in affected brain regions of SCA2 patients $[42,58]$. Accordingly, in our study, we observed astrogliosis and microgliosis in the mutant ataxin- 2 expressing hemisphere, which worsened during disease progression. Therefore, our observations also support the inflammatory response as a potential target to treat this condition. Overall, our data suggests that the striatal lentiviral mouse model is a robust and fast disease progression model, reproducing pathological hallmarks present in SCA2 patients.

In polyQ diseases, the causative expanded proteins tend to aggregate and assemble in large aggregates, recruiting other components including RNA-binding proteins, protein chaperones 
or transcription factors $[9,59]$, a process that is exacerbated by the failure in protein degradation systems, such as autophagy [60]. Previously, Wardman and colleagues showed that mutant ataxin- 2 is eliminated by autophagy rather than by the proteasome system [61]. Taken this in consideration, in our study we evaluated the autophagic pathway status in SCA2. The expression of expandedpolyQ ataxin-2 in neuronal cells and mouse striatum resulted in increased levels of the receptor SQSTM1, while its MRNA levels were upregulated in the SCA2 lentiviral mouse model. These results are in agreement with previous studies, which also demonstrated SQSTM1 abnormal accumulation in SCA2 transgenic mouse models [18, 62]. These results suggest ineffective lysosomal degradation, as SQSTM1 is also recruited to autophagosomes and is normally cleared at the final steps of the process [63]. Moreover, we found that LC3B-II, which is required for autophagosome formation, is decreased in cells expressing mutant ataxin-2, also supporting an autophagy dysregulation. Although LC3B-II levels were found to be upregulated in SCA2 transgenic mouse models, we observed no differences in the SCA2 lentiviral mouse model $[18,62]$. As autophagy is a very dynamic process, both decreased or increased LC3B-II levels could suggest autophagic impairment, therefore other autophagic markers should be also taken into consideration [64]. Additionally, the mRNA levels of the anti-apoptotic factor $\mathrm{BCl} 2$ were upregulated in the SCA2 lentiviral mouse model. Bcl2 binds to Beclin-1 protein, inhibiting autophagy initiation [65]. In this study, we also showed evidence for autophagy dysfunction in the brain of SCA2 patients. The impairment of autophagy is strengthened by an abnormal accumulation of autophagic markers in human SCA2 cerebellum and striatum, which agrees with observations in other neurodegenerative diseases. Altogether, these results suggests that mutant ataxin-2 could impact autophagy, thus exacerbating the accumulation of misfolded proteins in SCA2 disorder.

This work reinforces the crucial role of autophagy in SCA2, which could be a potential target for a therapeutic strategy. In fact, a study using an SCA2 cellular model reported that enhancing autophagy resulted in apoptosis decrease [61]. Here, we tested a pharmacological approach to induce the autophagic pathway in SCA2 cellular and mouse models. Previously, we have shown that cordycepin has the ability to improve several neuropathological and behavior deficits in MJD mouse models [65]. In the present study we also observed a neuroprotective effect using a long-term cordycepin treatment in the SCA2 lentiviral mouse model, resulting in decreased mutant ataxin-2 aggregation, preservation of the neuronal loss and reduced cell death.

In conclusion, in this study we developed a new mouse model that bear resemblance to some of the neuropathological hallmarks observed in SCA2 patients, thus constituting a useful model to study disease mechanisms and therapeutic strategies. Importantly, we provide evidence for the dysregulation of the autophagic pathway, in mouse models and SCA2 patients brain samples, and that autophagy upregulation could be a potential therapeutic target for SCA2.

\section{DATA AVAILABILITY}

The datasets used and/or analyzed during the current study are available from the corresponding author on reasonable request.

\section{REFERENCES}

1. Wadia NH, Swami RK. A new form of heredo-familial spinocerebellar degeneration with slow eye movements (nine families). Brain. 1971;94:359-74.

2. Orozco G, Estrada R, Perry TL, Araña J, Fernandez R, Gonzalez-Quevedo A, et al. Dominantly inherited olivopontocerebellar atrophy from eastern Cuba. Clinical, neuropathological, and biochemical findings. J Neurol Sci. 1989;93:37-50.

3. Pulst SM, Nechiporuk A, Nechiporuk T, Gispert S, Chen XN, Lopes-Cendes I, et al. Moderate expansion of a normally biallelic trinucleotide repeat in spinooerebellar ataxia type. Nat Genet. 1996;14:269-76.
4. Sanpei K, Takano H, Igarashi S, Sato T, Oyake M, Sasaki $H$, et al. Identification of the spinocerebellar ataxia type 2 gene using a direct identification of repeat expansion and cloning technique, DIRECT. Nat Genet. 1996;14:277-84.

5. Lastres-Becker I, Rüb U, Auburger G. Spinocerebellar ataxia 2 (SCA2). Cerebellum 2008;7:115-24.

6. Seidel K, Siswanto S, Brunt ERP, Den Dunnen W, Korf HW, Rüb U. Brain pathology of spinocerebellar ataxias. Acta Neuropathol. 2012;124:1-21.

7. Magaña JJ, Velázquez-Pérez L, Cisneros B. Spinocerebellar ataxia type 2: Clinical presentation, molecular mechanisms, and therapeutic perspectives. Mol Neurobiol 2013;47:90-104.

8. Stezin A, Venkatesh SD, Thennarasu K, Purushottam M, Jain S, Yadav R, et al. Nonataxic manifestations of Spinocerebellar ataxia-2, their determinants and predictors. J Neurol Sci. 2018;394:14-18.

9. Takeuchi T, Nagai Y Protein misfolding and aggregation as a therapeutic target for polyglutamine diseases. Brain Sci. 2017; 7. https://doi.org/10.3390/brainsci7100128.

10. Wen J, Scoles DR, Facelli JC. Effects of the enlargement of polyglutamine segments on the structure and folding of ataxin-2 and ataxin-3 proteins. J Biomol Struct Dyn. 2017;35:504-19.

11. Seidel K, Siswanto S, Fredrich M, Bouzrou M, den Dunnen WFA, Özerden I, et al. On the distribution of intranuclear and cytoplasmic aggregates in the brainstem of patients with spinocerebellar ataxia type 2 and 3. Brain Pathol. 2017;27:345-55.

12. Egorova PA, Bezprozvanny IB. Molecular mechanisms and therapeutics for spinocerebellar ataxia type 2. Neurotherapeutics. 2019;16:1050-73.

13. Cortes CJ, La Spada AR. Autophagy in polyglutamine disease: Imposing order on disorder or contributing to the chaos? Mol Cell Neurosci 2015;66:53-61.

14. Nascimento-Ferreira I, Santos-Ferreira T, Sousa-Ferreira L, Auregan G, Onofre I, Alves $S$, et al. Overexpression of the autophagic beclin-1 protein clears mutant ataxin-3 and alleviates Machado-Joseph disease. Brain. 2011;134:1400-15.

15. Fujikake N, Shin $M$, Shimizu S Association between autophagy and neurodegenerative diseases. Front. Neurosci. 2018; 12. https://doi.org/10.3389/fnins.2018.00255.

16. Marcelo A, Brito F, Carmo-Silva S, Matos CA, Alves-Cruzeiro J, Vasconcelos-Ferreira $A$ et al. Cordycepin activates autophagy through AMPK phosphorylation to reduce abnormalities in Machado-Joseph disease models. Hum Mol Genet 2018. https://doi.org/10.1093/hmg/ddy328.

17. Puorro G, Marsili A, Sapone F, Pane C, De Rosa A, Peluso S, et al. Peripheral markers of autophagy in polyglutamine diseases. Neurol Sci. 2018;39:149-52.

18. Paul S, Dansithong W, Figueroa KP, Scoles DR, Pulst SM Staufen 1 links RNA stress granules and autophagy in a model of neurodegeneration. Nat Commun 2018; 9. https://doi.org/10.1038/s41467-018-06041-3.

19. Pereira de Almeida L, Zala D, Aebischer P, Déglon N. Neuroprotective effect of a CNTF-expressing lentiviral vector in the quinolinic acid rat model of Huntington's disease. Neurobiol Dis. 2001;8:433-46.

20. Huynh DP, Yang HT, Vakharia H, Nguyen D, Pulst SM. Expansion of the polyQ repeat in ataxin-2 alters its Golgi localization, disrupts the Golgi complex and causes cell death. Hum Mol Genet. 2003;12:1485-96.

21. Kimura $S$, Noda T, Yoshimori T. Dissection of the autophagosome maturation process by a novel reporter protein, tandem fluorescent-tagged LC3. Autophagy. 2007;3:452-60.

22. Mendonça LS, Nóbrega C, Hirai H, Kaspar BK, Pereira de Almeida L. Transplantation of cerebellar neural stem cells improves motor coordination and neuropathology in Machado-Joseph disease mice. Brain. 2015;138:320-35.

23. Nóbrega C, Carmo-Silva S, Albuquerque D, Vasconcelos-Ferreira A, Vijayakumar U-G, Mendonça $\mathrm{L}$, et al. Re-establishing ataxin-2 downregulates translation of mutant ataxin-3 and alleviates Machado-Joseph disease. Brain. 2015;138:3537-54.

24. Nóbrega C, Nascimento-Ferreira I, Onofre I, Albuquerque D, Conceição M, Déglon $\mathrm{N}$, et al. Overexpression of mutant ataxin-3 in mouse cerebellum induces ataxia and cerebellar neuropathology. Cerebellum. 2013;12:441-55.

25. Nóbrega C, Nascimento-Ferreira I, Onofre I, Albuquerque D, Déglon N, Pereira de Almeida L. RNA interference mitigates motor and neuropathological deficits in a cerebellar mouse model of Machado-Joseph disease. PLoS One. 2014;9:e100086.

26. Hjorth JJJ, Kozlov A, Carannante I, Frost Nylén J, Lindroos R, Johansson Y, et al. The microcircuits of striatum in silico. Proc Natl Acad Sci USA. 2020;117:9554-65.

27. Alves-Cruzeiro JMDC, Mendonça L, Pereira de Almeida L, Nóbrega C. Motor dysfunctions and neuropathology in mouse models of spinocerebellar ataxia type 2: a comprehensive review. Front Neurosci 2016;10. https://doi.org/10.3389/ fnins.2016.00572

28. De Almeida LP, Ross CA, Zala D, Aebischer P, Déglon N. Lentiviral-mediated delivery of mutant huntingtin in the striatum of rats induces a selective neuropathology modulated by polyglutamine repeat size, huntingtin expression levels, and protein length. J Neurosci. 2002;22:3473-83.

29. Alves S, Régulier E, Nascimento-Ferreira I, Hassig R, Dufour N, Koeppen A, et al. Striatal and nigral pathology in a lentiviral rat model of Machado-Joseph disease. Hum Mol Genet. 2008;17:2071-83.

30. Koyano S, Yagishita S, Kuroiwa Y, Tanaka F, Uchihara T. Neuropathological staging of spinocerebellar ataxia type 2 by semiquantitative 1C2-positive neuron typing. 
Nuclear translocation of cytoplasmic 1C2 underlies disease progression of spinocerebellar ataxia type 2. Brain Pathol. 2014;24:599-606.

31. Estrada R, Galarraga J, Orozco G, Nodarse A, Auburger G. Spinocerebellar ataxia 2 (SCA2): Morphometric analyses in 11 autopsies. Acta Neuropathol. 1999;97:306-10.

32. Nóbrega C, Codêsso JM, Mendonça L, Pereira De Almeida L. RNA interference therapy for machado-joseph disease: long-term safety profile of lentiviral vectors encoding short hairpin RNAs targeting mutant ataxin-3. Hum Gene Ther. 2019;30:841-54.

33. Koyano S, Uchihara T, Fujigasaki H, Nakamura A, Yagishita S, Iwabuchi K. Neuronal intranuclear inclusions in spinocerebellar ataxia type 2: Triple-labeling immunofluorescent study. Neurosci Lett. 1999;273:117-20.

34. Pang JT, Giunti $P$, Chamberlain S, An SF, Vitaliani R, Scaravilli T, et al. Neuronal intranuclear inclusions in SCA2: A genetic, morphological and immunohistochemical study of two cases. Brain. 2002;125:656-63.

35. O'Callaghan $C$, Bertoux $M$, Hornberger $M$. Beyond and below the cortex: the contribution of striatal dysfunction to cognition and behaviour in neurodegeneration. J Neurol Neurosurg Psychiatry 2014;85:371-8.

36. Tanaka S, Young JW, Halberstadt AL, Masten VL, Geyer MA. Four factors underlying mouse behavior in an open field. Behav Brain Res. 2012;233:55-61.

37. Mcllwain DR, Berger T, Mak TW. Caspase functions in cell death and disease. Cold Spring Harb Perspect Biol. 2013;5:1-28.

38. Soriano E, Del Rio JA, Auladell C. Characterization of the phenotype and birthdates of pyknotic dead cells in the nervous system by a combination of DNA staining and immunohistochemistry for 5'-bromodeoxyuridine and neural antigens. J Histochem Cytochem. 1993;41:819-27.

39. Olejniczak M, Urbanek MO, Krzyzosiak WJ. The role of the immune system in triplet repeat expansion diseases. Mediators Inflamm 2015;2015. https://doi.org/ 10.1155/2015/873860.

40. Hong H, Kim BS, Im H-I. Pathophysiological role of neuroinflammation in neurodegenerative diseases and psychiatric disorders. Int Neurourol J 2016;20:S2-S7.

41. Huynh DP, Del Bigio MR, Ho DH, Pulst SM. Expression of ataxin-2 in brains from normal individuals and patients with Alzheimer's disease and spinocerebellar ataxia 2. Ann Neurol. 1999;45:232-41.

42. Hoche F, Seidel K, Brunt ER, Auburger G, Schöls L, Bürk K, et al. Involvement of the auditory brainstem system in spinocerebellar ataxia type 2 (SCA2), type 3 (SCA3) and type 7 (SCA7). Neuropathol Appl Neurobiol. 2008;34:479-91.

43. Katsuragi $Y$, Ichimura $Y$, Komatsu M. P62/SQSTM1 functions as a signaling hub and an autophagy adaptor. FEBS J. 2015;282:4672-8.

44. Lee YK, Lee JA. Role of the mammalian ATG8/LC3 family in autophagy: differential and compensatory roles in the spatiotemporal regulation of autophagy. BMB Rep. 2016;49:424-30.

45. Stavoe AKH, Holzbaur ELF. Autophagy in neurons. Annu Rev Cell Dev Biol 2019;35:477-500.

46. Satterfield TF, Pallanck L. Ataxin-2 and its drosophila homolog, ATX2, physically assemble with polyribosomes. Hum Mol Genet. 2006;15:2523-32.

47. Kiehl TR, Shibata $H$, Pulst SM. The ortholog of human ataxin- 2 is essential for early embryonic patterning in C. elegans. J Mol Neurosci. 2000;15:231-41.

48. Huynh DP, Figueroa K, Hoang N, Pulst S-M. Nuclear localization or inclusion body formation of ataxin-2 are not necessary for SCA2 pathogenesis in mouse or human. Nat Genet. 2000;26:44-50.

49. Aguiar J, Fernández J, Aguilar A, Mendoza Y, Vázquez M, Suárez J, et al. Ubiquitous expression of human SCA2 gene under the regulation of the SCA2 self promoter cause specific Purkinje cell degeneration in transgenic mice. Neurosci Lett. 2006;392:202-6.

50. Damrath E, Heck MV, Gispert S, Azizov M, Nowock J, Seifried C, et al. ATXN2CAG42 sequesters PABPC1 into insolubility and induces FBXW8 in cerebellum of old ataxic knock-in mice. PLoS Genet. 2012;8:e1002920.

51. Hansen ST, Meera P, Otis TS, Pulst SM. Changes in Purkinje cell firing and gene expression precede behavioral pathology in a mouse model of SCA2. Hum Mol Genet. 2013;22:271-83.

52. Dansithong W, Paul S, Figueroa KP, Rinehart MD, Wiest S, Pflieger LT, et al. Ataxin2 regulates RGS8 translation in a new BAC-SCA2 transgenic mouse model. PLOS Genet. 2015;11:e1005182.

53. Sen N-E, Canet-Pons J, Halbach MV, Arsovic A, Pilatus U, Chae W-H, et al. Generation of an Atxn2-CAG100 knock-in mouse reveals $\mathrm{N}$-acetylaspartate production deficit due to early Nat8l dysregulation. Neurobiol Dis. 2019;132:104559.

54. Varrone A, Salvatore E, De Michele G, Barone P, Sansone V, Pellecchia MT, et al. Reduced striatal [123I]FP-CIT binding in SCA2 patients without Parkinsonism. Ann Neurol. 2004;55:426-30.

55. Pirker W, Back C, Gerschlager W, Laccone F, Alesch F. Chronic thalamic stimulation in a patient with spinocerebellar ataxia type 2. Mov Disord. 2003;18:222-5.

56. Zala D, Bensadoun JC, De Almeida LP, Leavitt BR, Gutekunst CA, Aebischer P, et al. Long-term lentiviral-mediated expression of ciliary neurotrophic factor in the striatum of Huntington's disease transgenic mice. Exp Neurol. 2004;185:26-35.
57. Fakhoury M Microglia and astrocytes in Alzheimer's disease: implications for therapy. Curr Neuropharmacol. 2017;15. https://doi.org/10.2174/1570159x156661 70720095240.

58. Gierga K, Bürk K, Bauer M, Orozco Diaz G, Auburger G, Schultz C, et al. Involvement of the cranial nerves and their nuclei in spinocerebellar ataxia type 2 (SCA2). Acta Neuropathol. 2005;109:617-31.

59. La Spada AR, Taylor JP. Repeat expansion disease: progress and puzzles in disease pathogenesis. Nat Rev Genet 2010;11:247-58.

60. Vilchez D, Saez I, Dillin A. The role of protein clearance mechanisms in organismal ageing and age-related diseases. Nat Commun 2014;5:1-13.

61. Wardman JH, Henriksen EE, Marthaler AG, Nielsen JE, Nielsen TT. Enhancement of autophagy and solubilization of ataxin-2 alleviate apoptosis in spinocerebellar ataxia type 2 patient cells. Cerebellum. 2020;19:165-81.

62. Scoles DR, Dansithong W, Pflieger LT, Paul S, Gandelman M, Figueroa KP, et al. ALS-associated genes in SCA2 mouse spinal cord transcriptomes. Hum Mol Genet. 2020;29:1658-72.

63. Bjørkøy G, Lamark T, Brech A, Outzen H, Perander M, Øvervatn A, et al. p62/ SQSTM1 forms protein aggregates degraded by autophagy and has a protective effect on huntingtin-induced cell death. J Cell Biol. 2005;171:603-14.

64. Klionsky DJ, Abdel-Aziz AK, Abdelfatah S, Abdellatif M, Abdoli A, Abel S, et al. Guidelines for the use and interpretation of assays for monitoring autophagy (4th edition) 1. Autophagy. 2021;17:1-382.

65. Pattingre S, Tassa A, Qu X, Garuti R, Xiao HL, Mizushima N, et al. Bcl-2 antiapoptotic proteins inhibit Beclin 1-dependent autophagy. Cell. 2005;122:927-39.

\section{ACKNOWLEDGEMENTS}

We acknowledge the Light Microscopy Unit of ABC-RI, which is partially supported by national Portuguese funding FCT: PPBI-POCI-01-0145-FEDER-022122. We also thank Dr Claudia Florindo of Andor Technology for working with us and providing an opportunity to capture images on the new benchtop confocal. We also thank to the Center for Neuroscience and Cell Biology Microscopy unit. We extended our thank to the Município de Loule for the support in the publication. This study was funded by the Portuguese Science and Technology Foundation (FCT) project (ALG-01-0145FEDER-29480) "SeGrPolyQ", with CRESC ALGARVE 2020 cofunding, the French Muscular Dystrophy Association (AFM-Téléthon) project \#22424 and by the Ataxia UK (ZUNIALGA project). AM is supported by a Ph.D. fellowship from FCT (SFRH/BD/ 133192/2017).

\section{AUTHOR CONTRIBUTIONS}

AM performed the experiments and analyses and wrote the manuscript, ITA, RAR, and RGC performed some experiments, AR, JAC, BF performed several experiments and analysis, DVCB, CM performed some experiments and provided and critical review on the manuscript, $\mathrm{CH}, \mathrm{RJN}$, performed some experiments, LPA reviewed the paper, CN performed study concept and design and reviewed the paper. All authors read and approved the final paper.

\section{COMPETING INTERESTS}

The authors declare no competing interests.

\section{ETHICS APPROVAL AND CONSENT TO PARTICIPATE}

The study obtained the proper ethics consent for the work with animals as detailed in the material and methods section.

\section{ADDITIONAL INFORMATION}

Supplementary information The online version contains supplementary material available at https://doi.org/10.1038/s41419-021-04404-1.

Correspondence and requests for materials should be addressed to Clévio Nóbrega.

Reprints and permission information is available at http://www.nature.com/ reprints

Publisher's note Springer Nature remains neutral with regard to jurisdictional claims in published maps and institutional affiliations. 
Open Access This article is licensed under a Creative Commons At c) Atribution 4.0 International License, which permits use, sharing,
adaptation, distribution and reproduction in any medium or format, as long as you give appropriate credit to the original author(s) and the source, provide a link to the Creative Commons license, and indicate if changes were made. The images or other third party material in this article are included in the article's Creative Commons license, unless indicated otherwise in a credit line to the material. If material is not included in the article's Creative Commons license and your intended use is not permitted by statutory regulation or exceeds the permitted use, you will need to obtain permission directly from the copyright holder. To view a copy of this license, visit http://creativecommons. org/licenses/by/4.0/.

(c) The Author(s) 2021 